\title{
Operational restrictions on morphing of quasi- geometric 4D physical spaces
}

\author{
Jakub Czajko \\ Science/Mathematics Education Department, Southern University and A\&M College, \\ Baton Rouge, LA 70813, USA \\ E-mail address: sunswing77@netscape.net
}

\begin{abstract}
Since a hierarchical notion of dimension is needed to ensure that a virtual, indirect orthogonality of dimensions is maintained in higher-dimensional spatial structures, a generic function for furling and unfurling of the fourth dimension in four-dimensional (4D) spatial structures is proposed. The furling allows three extra dimensions (above the regular three) in a 6D algebraic structure to be represented as a single fourth dimension and thus effectively facilitates morphing of the 4D spacetime into its dual 4D timespace. The effect of the furling of the extra three dimensions resembles that of compactification proposed by Kaluza-Klein theory yet without curling of the furled dimension. The furling supports reexpansion of stringy space into yet another dimension and so enables mapping of radius $\mathrm{R}$ (of a closed string being squeezed beyond its minimal radius) into an inverse radius $1 / \mathrm{R}$, which was attributed to string duality, but is shown as due to duality of the $4 \mathrm{D}$ spatial structures of spacetime and timespace. Mathematically, it may appear as if further squeezing of the minimal string morphs it into an expanding pointletlike energy bubble so that the stringy spacetime reexpands in a new direction/dimension located within the bubbly dual timespace. So vibrating string is a mirror image of an energy bubblet, both of which do represent the same stringlet. By analogy, particle cast in spacetime could appear mathematically as having a mirror image (or its superpartner) cast in the dual timespace.
\end{abstract}

Keywords: Furling of higher dimensions; virtual orthogonality; multispatial hyperspace

\section{INTRODUCTION}

Abel and Galois have proved that polynomial equations (as algebraic prototypes of abstract geometric dimension) of degrees higher than 4 are insolvable in general [1]. The insolvability posted operational restriction on maximal degree of polynomial equations. It constrains thus also the number of abstract dimensions that can be directly represented in single geometric or quasi-geometric spatial structure. This is because constructability of such structures depends on the operational procedures that correspond to the structures. If a structural equation is insolvable in general, then the object it describes cannot be always unambiguously constructed [1].

Impossibility of construction means that the structure depicted by the structural equation cannot exist (i.e. it cannot be constructed) as a single standalone object and so it actually describes a conglomerate of certain simpler objects. 
Insolvable structural equations should be so rearranged as to make them solvable, of course. Yet the insolvability also indicates a deeper conceptual problem, namely that the former mathematics that ignored the insolvability can be unreliable if not outright faulty.

Because structures and their operational procedures always come hand in hand, the insolvability implies impossibility of constructing higher-dimensional (HD) structures by an analogy to single 3D spaces. The impossibility, in turn, means that yet another way of constructing geometric HD structures is needed [1]. Weyl wrote that multidimensional sets are built through - what he called - mathematical process [2], which is indicative of an arbitrary way of unrestrained mathematical thinking applied to invent basic notions.

The insolvability is not unsurmountable obstacle whose very presence needs to be tacitly concealed, as it was in former mathematics, but it is an indication that the former mathematics, which ignored hints supplied by previously unanticipated experiments, was rather unrealistic (and sometimes even plainly wrong) in its previous approach to issues of dimensionality and spatiality in general. Mathematics disassociated from experimental suggestions often created not only nonexistent yet possible worlds, but also some whose implementation in the actually existing physical reality is not operationally feasible. Pure mathematics did create - in Watson's words - fake worlds [3], even without violating any rules, i.e. when it was proceeding "by the book", so to say. Some authors suggested that spaces other than $(3+1) \mathrm{D}$ might correspond to dead worlds [4]. Nonetheless, HD spatial structures (as opposed to 3D single spaces) can be constructed in the context of an abstract multispatial hyperspace ( $\mathrm{MH})$, which - as a compound spatial structure of higher order and greater complexity - is conceptually different from any 3D single space [1].

Hence an insolvable structural equation should be recast in the $\mathrm{MH}$ in order for it to become solvable, at least in principle, and thus specify a constructible object. Otherwise, inferences based upon an insolvable structural equation can lead to misconceptions.

Since the physical world we live in is already established and seems to be fully, if not perfectly, operational (i.e. it has been meaningfully constructed) and there exist many quite independently varying distinct magnitudes (i.e. pure abstract dimensions), a reliable way to operationally admissible implementation of multidimensionality already exists in the Nature. Yet the simplistic inference that (because allegedly there is no reason to limit the number of independently varying parameters) the number of dimensions a space can have is also unlimited [5], is not admissible. Only the number of quasi-geometric spaces and spatial structures is unlimited and so is the total number of dimensions in the whole physical reality, but the number of dimensions within a single space is surely limited by feasibility of the operations that can be performed on objects depicted within the space, which - according to insolvability of polynomials of degree higher than $4-$ is apparently limited to just 4 , per proofs devised by Abel and Galois [1]. One may view dimension as an order of a matrix of vectors [6] or perhaps - equivalently - as that of systems of linear equations [7]. Since Grassmann and Cayley invented higher than 3D conceptual spaces, abstract geometry has lost its connection to geometric 3D single space [8]. The argument that HD geometries are thinkable [9] does not guarantee that HD objects are constructible by the very same traditional mathematical methods that have been developed for simple geometric objects fully immersed in geometric 3D single spaces.

Hence it is up to a (new) synthetic mathematics to discover and/or synthesize actual meaning of dimensionality and spatiality, preferably by taking cues from hints supplied by unanticipated results of curious experiments. Instead of inventing an artificial reality whose existence is then usually just postulated, we should learn about attributes of our abstract geometrical concepts from the various ways the Nature operates on them [10]. 
However, synthesizing new concepts (or perhaps just enhancements to some already established notions) from strange experimental hints requires that one should renounce making any existential postulates, regardless of whether they are made explicitly or just tacitly disguised as definitions. For postulating existence creates nonexistent artificial reality and so it effectively defeats the very purpose of the prospective syntheses. Since all former geometrical theories are actually conventional (according to Poincaré), their abstract axioms are just definitions in disguise [11] p. 50, [12] p. 293.

Operational completeness of legitimate procedures should ensure the feasibility of an actual construction of the geometric structures that correspond to their (valid) operational procedures. Anything else may turn into an artificial world that could exist only on paper, no matter how persuasively its existence might be postulated. For mathematical theorems are only formally true but not always logically true, because their truth is not independent of any interpretation or regardless of how the world was constructed [13], for proofs of theorems depend on axioms and primitive notions, the meaning of which relies on current interpretations of the paradigms that were accepted as true or just self-evident.

Yet because usually there are numerous ways to perform a compound operation with multiple hierarchically arranged stages that depend on selected sets of parameters and variables representing independently varying magnitudes, experimental hints can provide valuable cues as to which ways are preferred by the Nature for each set of circumstances. Since we do not always know right up front all the parameters and variables involved in the given set of circumstances that defines the actual situation, the Nature can show us (via strange experiment results) what is really needed for the (actually implemented) operations to succeed in the given situation before we can fully define it. Note that any predefined situation could unintentionally fix (or even eliminate) some of its features.

Case in point: former mathematics allowed operational nonsenses to be perpetuated for centuries [14] even though it has developed proven evidence that could have refuted at least some of them, were it not for the fact that experiments (including valid proofs as mathematical counterparts of physical experiments) have been commonly disregarded in the past. Inconvenient evidence was routinely ignored in mathematics (if their proofs cast a shadow of doubt on the validity of previously accepted pure-mathematical axioms or on our espoused paradigms, no matter how unrealistic the preconceived mathematical ideas might be). And in physics too, if unanticipated results of experiments contradicted some man-made ideas about the Nature and the ways it is allegedly supposed to work [15].

The tacitly forgotten inconvenient (though proven) achievements of Abel, Galois and Lagrange is yet another example of rather surreal approach to previously recognized (but unanticipated and thus difficult to accept by their successors) mathematical results [1]. It is possible to postulate existence of certain attributes admissible for one object/situation and then tacitly (yet often quite inadvertently) carry over these attributes onto a different object/situation, where the same attributes may not be appropriate. This could be done by such innocuous statement as "let a group $G$ generated by $r$ independent infinitesimal transformations be given [hence: exists]" [16], which can pertain to an abstract 'space' of certain groups [17], and then apply the theorems proven for such abstract groups also to some less abstract geometric objects whose operational structures are entirely different [16]. What is admissible for very abstract algebraic objects is not always appropriate for more restrictive geometrical objects, whose construction can be rigorously constrained.

The need to rectify scientific misconceptions (as opposed to often silly unscientific ones, whose origin could usually be traced to students) is prompted by past theoretical developments, some of which stumbled upon inadequate tools of former mathematics. An 
example which just cannot be ignored any longer is the curious abstract string duality that apparently causes reexpansion of the underlying stringy space into yet another distinct dimension when closed string is being squeezed beyond its minimal radius $[18,19]$. Note that the peculiar reexpansion is not contingent upon one's acceptance of the string theories. It is an issue related to singularity in general and to duality of abstract spatial representations of geometric objects in particular. For the reexpansion resembles similar curious behavior of Lagrange resolvents [1], which emerged prior to string theories.

Since spatial reexpansion is a structural morphing [1], topology could not address the issue [20]. For the spatial morphing involves dual representations of $(3+1) \mathrm{D}$ and $(1+3) \mathrm{D}$ spatial structures, which when taken together, add only one extra dimension though [21]. Although for the ease of devising abstract proofs separate spaces of tangent vector fields and normal vector fields were oftentimes considered [22], such a theoretical approach is not helpful for conceptual investigation of actual geometrical spaces. As a matter of fact, even Poincaré admitted that geometry of $3+$ dimensions is qualitatively different [23].

\section{SUMMARY OF OPERATIONAL RESTRICTIONS ON DIMENSIONALITY}

Since three-dimensional (3D) spaces cannot contain more than three unique mutually orthogonal generic directions (understood as the geometric dimensions that correspond to distinct, independently varying magnitudes), in order to maintain abstract orthogonality in higher-dimensional (HD) quasi-spatial structures one has to admit abstract multispatial hyperspace $(\mathrm{MH})$ comprising few hierarchically arranged 3D single spaces and their dual 3D spaces $[1,24,25]$.

Since every dual linear vector space (LVS) is orthogonal to its primary space, virtual orthogonality (between the dimensions of the primary and its dual space) is thus virtually established in the MH. For dimensions higher than $3^{\text {rd }}$ within the dual space, which is always orthogonal to the primary space, are orthogonal to all other dimensions by belonging to the dual space [1].

Hence virtual (indirect) orthogonality of higher than $3^{\text {rd }}$ dimensions is hierarchical. Higher dimensions are thus quantized in triples. Maintaining virtual orthogonality is indispensable for preservation of distances. Without this feature spatial structures would be pointless and as such meaningless [1].

When triple Cartesian product is viewed as threefold extended subspace $\left\{\mathbb{R}^{3}\right\}$ with its own, native length-vector basis $\left.\right|_{\mathbb{R}}$, it is equivalent to a tripartite function subspace $\mathbb{R}_{\mathbb{R}}^{3}[1]$

$\mathbb{R} \times \mathbb{R} \times \mathbb{R}=\left\{\mathbb{R}^{3}\right\}:=\mathbb{R}_{\mathbb{R}}^{3}$ and thus also $\left\{\mathbb{T}^{3}\right\}:=\mathbb{T}_{\mathbb{T}}^{3}$

The same must be true of $3 \mathrm{D}$ representation of temporal intervals $\left\{\mathrm{T}^{3}\right\}$ in their native vector basis $\left.\right|_{\mathbb{T}}$ because of duality of spatial and temporal representations. But in a foreign vector basis (wherein 3D curve could not be directly represented), the tripartite functions must be furled (dimensionally reduced) into an indirect 1D yet curvilinear representation:

$$
\mathbb{R}_{\mathbb{R}}^{3} \leftrightarrow \mathbb{R}_{\mathbb{T}}^{1} \text { and } \quad \mathbb{T}_{\mathbb{T}}^{3} \leftrightarrow \mathbb{T}_{\mathbb{R}}^{1}
$$

Conversely, this can be viewed as a spatial expansion of sorts too, when curve from dual space is unfurled (or transformed in reverse) from a foreign vector basis to its own native vector basis wherein it can be directly represented and thus appear as a composed 3D object. 
The same object could appear thus as unfurled 3D structure in its native vector basis and as furled 1D structure in the foreign vector basis that is dual to its native basis.

Nevertheless, the unfurled 3D curve that is directly representable in the vector space equipped with vector basis native to the curve can also be indirectly represented in a dual vector space equipped with vector basis that is foreign to the $3 \mathrm{D}$ curve if the $3 \mathrm{D}$ curve is furled into 1D representation, because the furled 1D curve is independent of the foreign vector basis. Furled representation is dual to its unfurled (primary) representation.

It was also shown in [1] that - due to duality of spatial and temmporal representations and the fact that elapsing time coordinate is not just superposed but also superimposed on all three length-based regular spatial coordinates - presence of 4D spacetime structure $\mathfrak{R}^{4}$ implies of necessity presence of its dual 4D abstract quasi-spatial timespace structure $\mathfrak{T}^{4}$

$$
\mathfrak{R}_{\mathbb{R}}^{4}=\mathbb{R}_{\mathbb{R}}^{3} \times \mathbb{T}_{\mathbb{R}}^{1} \Rightarrow \mathfrak{I}_{\mathbb{T}}^{4}=\mathbb{T}_{\mathbb{T}}^{3} \times \mathbb{R}_{\mathbb{T}}^{1}
$$

with time-related base vectors though. Hence furling and unfurling of curves overcomes the restriction on the maximal number of dimensions present within any single geometric 3D space posed by general insolvability of polynomials of degree higher than 4 .

Nevertheless, both spacetime and timespace are 4D quasi-spatial structures, not really regular geometric spaces like the Euclidean 3D single space. From the standpoint of the former single-space paradigm of physical reality one could also say that the spacetime is a sheaf of geodesics in a superspace, which itself is a stratified union of manifolds [26]. Note that in the former mathematics the connotation of the term 'space' was akin to 'set'. It could be confusing, because the term 'space' alluded to constructible geometric space.

\section{REPRESENTATIONS OF LINEAR OBJECTS IN 4D SPATIAL STRUCTURES}

In the 4D spacetime the elapsed time coordinate/subspace $\mathbb{T}^{1}$ is indirectly represented by $1 \mathrm{D}$ time line (or by time curve in general). Time interval is pictured in sppacetime as an imaginary length interval $\mathbb{T}_{\mathbb{R}}^{1}=$ ict where c denotes the speed of light in vacuum (or in the given medium) that is assumed as being universally or at least locally constant. In the usual length-based vector base $\left.\right|_{\mathbb{R}}$, which is foreign to time flow, the time line/curve interval cannot be represented directly and thus it must be furled. For it is only indirectly pictured there as an extra, imaginary (hence foreign) 1D length interval.

Relying on hints supplied by Lorentz's experiments Einstein got the idea of time (as extending the $3 \mathrm{D}$ single space into 4D spacetime) quite right, even though his special theory of relativity (STR) emerged before most recent mathematical theories of generic dimension came up. Yet elapsing time is not only extending the usual three length-based space (LBS), but it is superimposed on the latter too [1]. Therefore the STR (and then also Minkowski's) depiction of relationships existing between the LBS and intervals of time flow is not entirely correct. It clearly pertains to elapsed time, but it is valid only when the time interval is straightlinear, not for - curvilinear in general - flow of time.

Although we do not have clear picture of interdependence of these two mutually dual spatial structures of spacetime and timespace in mathematics yet, combining physical and mathematical evidence can allow us to uncover necessary features of these two abstract quasispatial structures. When taken alone, neither mathematics nor physics can describe them adequately enough to reveal all their attributes. But when combined, mathematical 
requirements of operational validity and physical hints paint pretty convincing picture of how these two mutually dual quasi-geometric spatial structures are interrelated, indeed.

Inferences by analogy are not substitutes for proofs, of course, but it is a good start to get a preliminary idea of what the interdependence of spacetime and timespace really is. The eqs. (3) show that only in their native vector bases the respective intervals of length and time flow can be represented directly (i.e. unfurled), whereas in their foreign vector bases, which are dual to their native bases, they can be depicted only indirectly (i.e. when furled). The nuanced conceptual distinction was not always explicitly recognized before, because - due to undue influence of mathematically challenged former physics - the 4D spacetime was previously considered as the only possible extension of the 3D LBS.

Despite clear prior mathematical evidence that $(3+1) \mathrm{D}$ and $(1+3) \mathrm{D}$ spatial structures are formally indistinguishable [21,20], both Einstein and Minkowski ignored the fact that a 4D spatial structure of timespace can exist as an extension of a certain (dual to the LBS) single 3D time-based space (TBS). This is not a critique of their achievements, but strong argument for truly integrated approach to teaching of mathematics and physics in parallel. Physics can guide mathematics by providing checks to otherwise unconstrained abstract mathematical thinking. When taught standalone, neither of these sciences can avoid inadvertent creation of scientific misconceptions.

\section{MAPPING RADIUS FROM LBS INTO INVERSE RADIUS FROM TBS}

In timespace, however, the distance coordinate/subspace $\mathbb{R}^{1}$ is indirectly represented by $1 \mathrm{D}$ distance line (or curved) interval $\mathbb{R}_{\mathbb{T}}^{1}$ cast in a certain time-based vector base $\left.\right|_{\mathbb{T}}$, which is foreign to distance intervals.

Just as for spacetime (wherein any time interval is indirectly pictured as an imaginary length interval $\mathbb{T}_{\mathbb{R}}^{1}=$ ict ), I conjecture thus that in timespace length interval is pictured indirectly in time versors as $\mathbb{R}_{\mathbb{T}}^{1}=\mathrm{ic} / \mathrm{R}$ where $\mathrm{R}$ is a radial, in general, distance interval.

Because the regular length-based distance intervals cannot be represented directly in a foreign time-based vector base, they are pictured indirectly as an extra, i.e. imaginary (or foreign) elapsed length/distance interval that must be furled as a $1 \mathrm{D}$ object therein. Note that this conjecture is not an existential postulate, but a statement of theoretical fact (that is supported by experimentally well confirmed special relativity). Its truth should also be confirmed by some other experiments. For now, however, we have only a mathematical result of reasoning from string theory (as a thought-experiment - hence a counterpart of physical experiment) to show that the conjecture is true indeed, at least in string theory.

From the relation (2) and the representation of radius within the timespace $\mathfrak{T}^{4}$ that is conjectured above we obtain raw mapping of the string radius $\mathrm{R}$ into its inverse radius $1 / \mathrm{R}$

$$
\mathbb{R}_{\mathbb{R}}^{3} \leftrightarrow \mathbb{R}_{\mathbb{T}}^{1} \quad \& \quad \mathbb{R}_{\mathbb{T}}^{1}=\frac{\text { ic }}{\mathrm{R}} \Rightarrow \mathrm{R} \rightarrow \frac{\mathrm{ic}}{\mathrm{R}}
$$

which was usually attributed to string duality [19] p.29, but mathematically it arises from the inherent duality of the twin 4D spatial structures of spacetime $\mathfrak{R}^{4}$ and timespace $\mathfrak{T}^{4}$.

The implication (4) suggests that these two spatial structures are intertwined like yin and yang, so that if a quasi-geometric object in the spacetime is shrinking then its dual image in the timespace is rising and vice versa. But unlike the yin-yang symbolic pair, the mutually dual abstract quasi-spatial structures of spacetime and timespace refer to the same 
pangeometric, and thus identical, physical entity. These two dual spatial structures are just inverted mirror images of each other that are superimposed upon each other.

The conjecture about furled distance intervals already gives us tentative answer to the question of why the radius of a closed string, when squeezed apparently beyond minimal radius, maps to an inverse radius [19] p. 29. Maybe squeezing the closed string beyond its minimal radius transforms it into a pointletlike (i.e. with nonzero size) energy bubble so that the stringy 4D spacetime apparently reexpands into yet another direction/dimension, which belongs to the dual 4D timespace.

The possibility of such a behavior was already hinted at by Lagrange resolvents [1]. But underpinnings of the spatial reexpansion needs the new synthetic mathematics to be seen for what it really is.

Notice that the mapping of the string radius $\mathrm{R}$ into its inverse radius $1 / \mathrm{R}$ depicted on the right-hand side (RHS) of the implication (4) involves imaginary potential energy $U$

$$
\mathrm{U}=\frac{\mathrm{ic}}{\mathrm{R}}=\mathrm{icV} \text { where } \mathrm{V}=\frac{1}{\mathrm{R}}
$$

of all surrounding center-bound force fields, such as gravitational and Coulomb field, for instance. During transition from spacetime to timespace the mapping ties the radius $\mathrm{R}$ to generic radial potential $\mathrm{V}(\mathrm{R})$ of the interaction between the fields and the vibrating string.

Because the formal mapping of radius into its inverse radius happens under influence of the fields within which the string vibrates, the transition that involves transformation of the vector basis of the spatial structure of spacetime into that of its dual structure of timespace is actually morphing also the squeezed minimal string into an energy bubble. It is not that the string becomes an energy bubble, for the string always is the energy bubble too and the morphing merely changes its representation. In the native vector base of the spacetime the (foreign to the spacetime) energy bubble was only indirectly represented by the string, whereas in the native vector base of the timespace the bubble is represented directly. But (foreign to the timespace) strings cannot be directly represented in the dual timespace and therefore they must morph into energy bubbles.

At this point an alert reader might rightly object: Since I have shown ([27,28]) that potential energy (as a counterpart of work done by the given field) can have three spatial components, how come it cannot be directly represented in spacetime whose three regular spatial axes are also length-based? Let us say potential energy $E$ of a local interaction has three spatial components $\mathrm{E}_{\mathrm{x}}, \mathrm{E}_{\mathrm{y}}, \mathrm{E}_{\mathrm{z}}$ spreading along the spatial axes $(\mathrm{X}, \mathrm{Y}, \mathrm{Z})$. This means that the potential energy $\mathrm{E}$ is only decomposed into directions of the axes and so it can be only indirectly represented by spatial intervals aligned to the coordinate axes.

The three spatial energy components mean that the energy $E$ has only been decomposed, not really actually composed from certain frequency-based components, in which case it could be represented directly therein. The necessity to distinguish between spatial composition and mere decomposition along spatial axes has already been emphasized by Riemann [29].

From this new mathematical point of view, vibrating strings depicted in 4D spacetime could appear as elongated counterparts of pointletlike "bubblets" when cast in terms of energy in 4D timespace. Hence - on a deeper level of inquiry - both vibrating strings and energy bubblets might be treated as two dual images of certain stringlets, by analogy to quantum duality of waves and particles.

This interpretation can also vindicate the idea of supersymmetry. But instead of looking for superpartners to particles in the 4D spacetime, one might consider abstract superpartners 
as dual representations of the regular particles, when the latter are recast in the 4D timespace. This venue shall be pursued elsewhere.

Hence all intervals could be consistently measured within each of these two mutually dual respective quasi-spatial structures: spacetime and timespace. They are represented in two distinct homogeneous coordinates systems or perhaps in a heterogeneous coordinate system with two dual vector bases, if you will. However, because these two, essentially logical, spatial structures of spacetime and timespace actually refer to exactly the same quasigeometric or pangeometric structure, we have to deal with 6 abstract dimensions, the set of three of which are always virtual. Here I am using the qualifier 'virtual' in its plain/basic English meaning of the word, not to be confused with that virtual dimension of abstract moduli space - see [30] p. 8, for instance. By the same token, however, I must distinguish between the geometric space and an abstract spatial or quasi-spatial structure, which is akin to set (or to just selection procedure operating on) point-sets.

Without recognizing its existence, modern pure mathematics is still trying to describe multispatial physical reality in terms of the former paradigm of single-space reality. The previous paradigm lacks proper words to describe the multispatial reality, and therefore - just by trying to be consistent - the former mathematics, often quite inadvertently, made some conceptual mistakes. That is why it perpetuated various tacitly concealed scientific misconceptions for many centuries while unwittingly allowing misinterpretations of some oversimplified experiments [31] and even endorsing fake theorem [14].

\section{ALGEBRAIC REPRESENTATIONS OF 4D SPATIAL STRUCTURES}

Formal equivalence of the $(3+1) \mathrm{D}$ and $(1+3) \mathrm{D}$ spatial structures of $4 \mathrm{D}$ spacetime and 4D timespace - in conjunction with furling of the usual three dimensions and unfurling of the fourth dimension - apparently can also imply presence of complex, abstract algebraic or 6D pangeometric hyperstructure $\mathfrak{S}$ with a heterogeneous complex $(3+3) \mathrm{D}$ vector base

$$
\mathfrak{R}_{\mathbb{R}}^{4} \Leftrightarrow \mathfrak{I}_{\mathbb{T}}^{4} \Rightarrow \mathfrak{R}_{\mathbb{R}}^{4}=\mathbb{R}_{\mathbb{R}}^{3} \times \mathbb{T}_{\mathbb{R}}^{1} \rightrightarrows \mathfrak{S}_{\mathbb{R} \rightarrow \mathbb{T}}^{6}:=\left\{\mathbb{R}^{3}\right\} \oplus \mathrm{i}\left\{\mathbb{T}^{3}\right\}=: \mathbb{R}_{\mathbb{R}}^{3} \bigoplus \mathrm{i} \mathbb{T}_{\mathbb{T}}^{3}
$$

which shows that, algebraically, these two 4D dual quasi-spatial structures can be treated also as an algebraic 6D structure comprising two dual 3D single spaces. However, one of the paired 3D single spaces should be taken as imaginary, because they have distinct and different vector bases and yet they are superposed in this setting (hence the 6D structure is additive: $\oplus$ ), wheras in the 4D spatial structure they are also superimposed upon each other (hence the Cartesian product sign: $\times$ was used therein).

Therefore this particular representation is required by their mutual duality in order to ensure both: virtual overall orthogonality [32] and solvability of prospective structural equations [1] of these two mutually immersed 4D spatial structures. For when a 3D single space has dual, the latter should be rendered as being an imaginary 3D space here in the hyperstructure $\mathfrak{S}$, because it cannot be directly represented in the vector base associated with the primary $3 \mathrm{D}$ single space. Actually these two 3D single spaces, namely: the $\mathbb{R}_{\mathbb{R}}^{3}$ (LBS) and $\mathbb{T}_{\mathbb{T}}^{3}$, which appears as a 3D time-based space (TBS) are just mutually dual, or inverted mirror images of each other. Both LBS and TBS represent the same underlying geometric 3D single space with two different, mutually dual vector bases though.

Cole has also realized that complex 4D spacetime may be represented by 6 real coordinates or 8 parameters [33] and so did few other authors [34,35]. Relations existing 
between the 3 spatial directions (i.e. real dimensions), 4 complex dimensions, 6 complex coordinates and 8 parameters shall be discussed elsewhere in terms of pangeometry.

Due to duality of the 3D spaces (LBS and TBS) immersed in the 6D hyperstructure $\mathfrak{S}$, reciprocity between space/length and time axes demands that each 3-tuple, i.e. three axes of the usual geometrical space and three axes of accumulated time flow, should be interchanged [36], [37]. Nevertheless, these axes of time flow in TBS actually refer to accumulated time rates rather than to elapsed time intervals [38]. Based upon physical considerations Lanciani has already concluded that spacetime should also have 6 distinct dimensions and that the two extra dimensions must be of time kind if electrodynamics is to be consistent [39].

Also Kar considered relativistic time variable as representing a time vector [40] and concluded that the $4^{\text {th }}$ coordinate of spacetime of spacetime is contravariant vector in the direction of time [41].

Tifft has investigated 3D temporal space with one radial and two lateral dimensions of time, where the radius of the temporal space replaces the standard radius of curvature in describing redshifts in spectra taken from stars in distant galaxies seen when photons transfer between objects on different timelines [42]. There is no doubt that geometry [of physical spaces] must be spatio-temporal [43], but it should also comply with operational and physical demands imposed on it by unquestionable physical relationships.

Since in its apparent 6D image the algebraic hyperstructure $\mathfrak{S}$ (which spans the two superimposed 4D spatial structures of spacetime and timespace) can also be viewed as comprising two different superposed single 3D vector spaces, namely LBS and TBS, each with different and distinct vector base though. As the $6 \mathrm{D}$ spatial hypertructure $\mathfrak{S}$ is a conglomerate of two distinct superposed dual 3D single spaces. Yet $\mathfrak{S}$ is also an overlap of two superimposed 4D spatial structures of spacetime and timespace.

Although the two 3D single spaces have also emerged as dual in the 6D notation, they add extra attributes (beside duality) to the 6D hyperspatial conglomerate $\mathfrak{S}$, which has two distinct and different 3D vector bases. Notice that 4D spacetime and timespace are geometric quasi-spatial structures, whereas the algebraic hyperstructure $\mathfrak{S}$ is an object not of geometric but of purely algebraic character, even though it could get assigned also a geometric interpretation - compare $[44,45]$. To explore algebraic input to geometry we need pangeometry, which is a theoretical umbrella to handle both: purely algebraic hyperstructures as well as their components (i.e. quasi-geometric spatial structures).

Although no 4th dimension can be pairwise orthogonal with the three ones of any of the 3D single spaces [1], it becomes globally orthogonal via virtual orthogonallity, i.e. by being qualified as belonging to the imaginary dual 3D single space wherein those dual dimensions are also pairwise orthogonal. Hence the two superposed and mutually dual 3D single spaces (each with its own native vector base that are superimposed within 4D structures) resemble space of bivectors, which could provide pretty good anology for grasping the idea of abstract HD spaces and quasi-geometric spatial structures. Recall that multivectors can also be considered as representing mixed rank tensors [46].

If the set of four distinct versors $\left\{\mathrm{e}_{0}, \mathrm{e}_{1}, \mathrm{e}_{2}, \mathrm{e}_{3}\right\}$ is taken as the basis of the regular 4D spacetime, then the 6 so-called volutors $\mathrm{e}_{0} \wedge \mathrm{e}_{1}, \mathrm{e}_{0} \wedge \mathrm{e}_{2}, \mathrm{e}_{0} \wedge \mathrm{e}_{3}, \mathrm{e}_{1} \wedge \mathrm{e}_{2}, \mathrm{e}_{2} \wedge \mathrm{e}_{3}, \mathrm{e}_{3} \wedge \mathrm{e}_{1}$ evidently form a 4-basis in the abstract space of bivectors (for the wedge product corresponds to generic imaginary vector product of vectors), and so it appears that both bivectors and the 4D spacetime structure is effectively 6 dimensional [47], even though they have only 4D vector bases. It is thus a conceptual departure from the original intuitive meaning of geometric dimensionality of regular 3D single spaces. It also severs most direct ties of such abstract dimensionality to coordinatization, even though it is formally correct. 
Coordinatization of $\mathrm{nD}$ manifold $\mathrm{M}$ means assigning a set of $\mathrm{n}$ real numbers ( $\mathrm{n}$-tuple) to each point of the manifold in such a way that to two distinct points of $\mathrm{M}$ correspond distinct n-tuples [48] p. 26. Furthermore, any concept that is invariant under the group of diffeomorphisms can be carried over to the manifold $\mathrm{M}$ in a way that does not depend on the choice of particular coordinate system for M [48] p. 29.

However, coordinatization does not always mean also metrization of the underlying manifold of the physical space [49], even though in the old conceptual framework of the former (yet still "reigning") paradigm of single-space physical reality we used to assume that unique distance between any two points can always be assigned, no matter what the dimensionality of the manifold was presumed to be, just because the points - according to the single-space reality paradigm - belong to the same underlying point-set space.

I am not saying that unique distance could not be assigned to arbitrary points located in HD structures. However, assigning distances on manifolds spanning HD multispatial structures is not as simple as Riemann stipulated, because such structures are hierarchical, not flat. Hence higher dimensions must be superimposed, not merely added/superposed.

Multistaged, hierarchical pangeometric reality evidently demands departure from the single-space reality paradigm of former mathematics and based on it physics.

At this point I owe the reader an explanation. Since we inherited splendid tradition of developing geometric algebra (GA) with successful applications of multivectors, why not just use it as the foundation for explaining both mathematical and physical reality. While the GA is resourceful in its own right, it cannot be applied to quantum mechanics because GA does not distingush between commutativity and noncommutativity.

For under projective equivalence all information about signs (hence orientations) is lost, but the meet and join operations on blades are commutative just as they are on sets [50]. Vector/cross and scalar/dot product of vectors/blades are dual [50,51] p. 107, but the rarely mentioned Grassmann's regressive product (also known as meet), which does pertain to both duality and to scalar product, also refers to the point of intersection of the pure vectors (blades/intervals) [50]. I shall show in a subsequent paper that this is just yet another indication (or an abstract mathematical hint, if you will) that algebraic operations actually point to presence of a certain abstract, quasi-geometric multispatial structure.

Disregarding biblical warning against pouring new wine into old wineskins, the GA tries to describe multispatial physical reality without mentioning multispatiality by name and thus also without words needed to describe all facets of the actual physical reality. Despite its ingenuity GA operations are restricted to the former paradigm of single-space reality. GA did not ask the question: what is the underlying actual reality it operates on?

It is easy to see that the $6 \mathrm{D}$ pangeometric hyperstructure $\sqrt{ }$ must come in two flavors too. While the eq. (6) shows hyperspatial expansion of the primary $3 \mathrm{D}$ single space that is equipped with length-based vector base into its dual 3D single space (equipped with a timebased vector base), reverse expansion can also be performed in the following way:

$$
\mathfrak{I}_{\mathbb{T}}^{4} \Leftrightarrow \mathfrak{R}_{\mathbb{R}}^{4} \Rightarrow \mathfrak{I}_{\mathbb{T}}^{4}=\mathbb{T}_{\mathbb{T}}^{3} \times \mathbb{R}_{\mathbb{T}}^{1} \rightrightarrows \mathfrak{S}_{\mathbb{T} \rightarrow \mathbb{R}}^{6}:=\left\{\mathbb{T}^{3}\right\} \oplus \mathrm{i}\left\{\mathbb{R}^{3}\right\}=: \mathbb{T}_{\mathbb{T}}^{3} \oplus \mathrm{i} \mathbb{R}_{\mathbb{R}}^{3}
$$

which is complex structure that is formally dual to that depicted by the chain of eqs. (6).

Notice that the hyperstructure $\mathfrak{S}$ standing on the right-hand side (RHS) of the chains of eqs. (6) and (7) - which was asumed as being a 4D quasi-spatial geometric structure, because it emerged from equivalence of two 4D spatial structures - is effectively also 6D algebraic structure comprising two dual 3D single spaces, each with its own native vector base. The 
apparent contradiction that two dual 4D spatial structures can be mapped onto a 6D structure is solved by unfurling of each of the fourth dimensions of the 4D structures.

The obvious duality of eqs. (6) and (7) also suggests that the 4D world is spherical with respect to the 3 regular spatial axes of the usual length-based 3D single space, but it also appears to be hyperboloidal with respect to the 1D elapsed time axis. This remark agrees with analogous conclusion reached by Kar and Dutta in [52].

Moreover, as Törnebohm already pointed out - if clocks and measuring rods behave (special-)relativistically by adjusting themselves to a field [even if the field is combined general-relativistic (i.e. radial gravitational) field and a special-relativistic field due to an artificial velocity], then both space and time in the combined spacetime structure are field concepts [53]. In such a case their underlying representation should be similar too.

While duality suggests that these 3D single spaces can be overlaid upon each other, which concurs with them being mutually superimposed [1], their formal equivalence - as suggested by the chain of eqs. (6) and (7) - is indicative of some hyperspatial connection whose presence (to the best of my knowledge) has never been explored, neither in linear algebra nor in geometries. Edward Kasner has already realized the need for $6 \mathrm{D}$ algebraic spatial structure to encapsulate/embed abstract 5D geometric structures, such as the one proposed in Kaluza-Klein theory [54]. However, the chains of eqs. (6) and (7) indicate that the $6 \mathrm{D}$ algebraic structure must not be real, but complex, if the operational procedure that corresponds to the structure is to be solvable, according to Abel and Galois [1].

When mutual (hence formally equivalent) duality of two 3D single geometric spaces accomodates two flavors of an algebraic hyperstructure $\mathfrak{S}$ spanning two 4D quasi-spatial structures, namely $(3+1) \mathrm{D}$ and $(1+3) \mathrm{D}$ ones, this suggests that a transition exists between these two, which seem to belong to a closed, intertwined hyperspatial system. For these two flavors are formally quite indistinguishable. From this point of view, the unfurling of 1D function/variable (that is cast in a foreign vector base) transforms it into its 3D dual counterpart, which is cast in its native vector basis. The process of dimensional unfurling could be viewed as dimensional reexpansion, if the corresponding to it process of furling is perceived as a dimensional reduction associated with dimensional contraction.

While the process of spatial contraction has no precedent in mathematics, its driving of dimensional reduction of one spatial structure and simultaneous reexpansion of its dual spatial structure resembles that curious behavior of Lagrange resolvents [1]. Therefore it should be handled by a pangeometry of multispatial hyperspaces. Its presence in natural phenomena is also indicated by spatial embeddings of some physical spaces.

\section{MULTIDIMENSIONAL FLOW OF TIME VERSUS SPACE OF TIME}

Some authors claim that multidimensional time implies existence of a space of time [55]. Nevertheless, mathematically it is not really admissible statement. The eq. (7) only shows that presence of a certain abstract multispatial hyperspace allows for fairly similar (though not really identical) dual representations of operational procedures (or processes such as accumulations) and corresponding to them generic quasi-geometric structures. In the sense, there seems to exist a geometric (or quasi-spatial) accumulation of rates of time flow [38], but no space of elapsed time. These topics shall be discussed more elsewhere.

The operational approach to exploration of issues of dimensionality and spatiality in the present note sprouted from ideas developed and proven by Abel, Galois and Lagrange [1]. 
Although proposals of multidimensional time flow were very valuable contributions, which did inspire much research, their mathematical expressions, usually made in terms of the elapsed-time variable $t$, are unacceptable, for models of multidimensional elapsed time must not yield more elapsing time than physical clocks actually measure. Time is superimposed on all the regular spatial dimensions [1]. Accumulation of elapsing time actually requires different quasi-geometric abstract structure of multispatial hyperspace (MH). Besides, even the 4D spacetime is an abstract spatial structure, not just space [1].

We should not merely extend the old notion of dimensionality of 3D simple spaces onto HD spatial structures without ensuring that the structures are operationally sound. Although one can always write structural equations in $\mathrm{n}$ coordinates by extension of the three coordinates, this act does not make such equations conceptually correct even if their values are admissible in most practical approximations. Conceptual validity of any formal expression/representation is indispensable for making meaningful inferences.

Any new theory of multidimensional time in $6 \mathrm{D}$ spacetime must be complementary to the ordinary 4-dimensional relativity, unifying new concepts and capable of explaining experimentally well-established facts [56]. Cole's approach with parallel time vectors in a frame is not sufficient to reproduce $\mathrm{R}^{4}$ from $\mathrm{R}^{6}$, however [57]. Although many of Cole's arguments are on target [58,59], his approach to multidimensionality of [elapsed] time creates problems [60]. Also his extension of Schwarzschild metric to 6 dimensions in terms of elapsed time gives wrong estimate for gravitating mass of ordinary matter [61]. Some authors argued that no present facts contradict multidimensionality of time [62], but such a statement could never be decisive because it is based upon negation of innumerable possibilities. While the idea of multidimensionality of time flow is exciting, its formal implementation in the former mathematics failed.

A 6D spacetime with signature $(-1,1,1,1,1,1)$ and two extra dimensions compactified on a manifold with a radius $\mathrm{R} \sim 1 \mathrm{~mm}$ was proposed in [63], but it was deemed unrealistic [64]. Operationally viable dimensional furling supersedes compactification.

Nonetheless, since events could be recorded by multiple clocks in terms of [elapsing] time [65], the idea of multidimensional accumulation of time (rather than elapsing time) should be explored, for multidimensionality of regular elapsing time is rather untenable. Previous mathematical approaches to multidimensionality within the former single-space reality paradigm failed to address the conceptual issues that crop up whenever nontrivial theoretical questions emerged. Yet when considered from the alternative standpoint of possibly multispatial structure of the physical reality we live in, formerly unresolved or unreconciled conceptual issues are qualitatively explainable. Hence also a conceptually new quantitative approach matching the successful qualitative one should be developed. However, the new approach should not be based upon preconceived ideas of what the mathematical underpinnings of the actual physical reality should look like, but rather on hints supplied by incontrovertible but formerly unanticipated experimental results. Let the Nature decide which mathematical concepts yield the best model of physical reality.

Traditionally, mathematics was used to reduce apparently unanswerable problems to simpler ones. While useful in approximations, the reductionist approach sets aside most conceptual issues, however. The example of Lagrange resolvents apparently growing more complicated when the number of dimensions exceeds four demonstrates that the overly simplistic reductionism has limits [1]. What seems more complicated is perhaps just an indication of much greater complexity of the concept of dimension. Mathematics should not keep on stubbornly ignoring the mathematical results that do not line up along its traditional line of thought, while avoiding inconvenient conceptual issues. 
Therefore multispatial approach should be tried, preferably with the use of the new synthetic mathematics that takes cues from hints of certain unbiased yet formerly quite unexpected experimental results, provided the syntheses avoid making any arbitrary existential postulates. For most existential postulates tend to create an artificial reality. We need new mathematical concepts which can model the actual physical reality.

Admittedly some aficionados of the idea of multidimensional time flow have already scored several points in its defense and so did also their critics in their attempts to detract the idea. With few exceptions both presented well-rounded arguments. However, because of its adherence to the old paradigm of single-space reality, former mathematics appears incapable to address the issues of higher dimensions in an operationally responsible way. The singlespace reality paradigm derailed many valid mathematical inferences.

The current mathematically-averse arbitrary paradigm of single-space reality cannot support multidimensional time flow. It has to be upgraded to a new paradigm that would admit presence of multispatial hyperspace with mathematically legitimate (i.e. at least operationally complete) structural rules of composition of such multispatial hyperspaces, which the former mathematics lacks. Hence again, mathematics needs an overarching umbrella of a new synthetic mathematics with pangeometry to achieve all that.

Yet embeddings of higher-dimensional spatial structures affirm the apparent need for the presence of multidimensional accumulation of time as well as hierarchically arranged realistic dimensions. Many celebrated mathematicians (such as Poincaré and Hilbert, to name just a few) disengaged mathematics from its former role as the queen of all exact sciences and apparently channeled mathematical explorations to what they could handle with ease. This resulted in arbitrary mathematical endeavors tailored to the whims of their creators, with almost statutory disregard for everything (even for some rigorously proven past mathematical results) that did not quite fit their arbitrary preconceived ideas about the actually existing physical reality. Mathematics should stop dictating what the Nature is supposed to look like and start investigating its actual modes of operation instead.

While abstract investigations are commendable even if these do not correspond yet to any known reality, disrespect for certain inconvenient mathematical truths (i.e. proven theorems) and sidelining or suppression of inconvenient yet mathematically legitimate alternatives makes such investigations pointless. These and other evasive trends made former mathematics unable to explain formerly unanticipated experimental results whose explanation required modification of the former single-space reality paradigm [27].

\section{EMBEDDINGS OF HIGHER-DIMENSIONAL SPATIAL STRUCTURES}

Thorne concluded that 6D hyperspace is needed to embed 3D curved space [66] and Edward Kasner has already found that proper embedding of 4D or 5D subspaces actually requires $6 \mathrm{D}$ algebraic space, which looks geometrically like a pair of 3D single spaces, based on symmetry and the sort of reasoning that aims at establishing both pairwise as well as global orthogonality of all abstract dimensions [67] p.104. He also showed that 4D Einstein manifold cannot be embedded within a 5D flat space [54] and that certain Einstein solutions can be immersed only in an abstract 7D flat space [67] p.105. He also demonstrated that solar gravitational field can only be properly embedded in an abstract $\mathrm{nD}$ structure where $\mathrm{n}$ falls in the range between 5 and $10(5 \geq n \leq 10)$ dimensions [68].

If embedding of the 5D Kaluza-Klein spacetime requires presence of certain two 3D single geometric spaces [67] that should act in unison as a tandem yet also independently of 
each other (i.e. in parallel but as a single pair too), the embedding might suggests a dual 'entanglement' of a pair of two distinct 3D images of the same 6D spatial structure. Hence dimensionality is not just an intrinsic feature of the underlying space itself, but seems to be ruled also by certain (pangeometric) symmetries external to the given space.

When investigating possible embedding functions, it was noticed that line elements in both Kasner embedding signature (++----) and Fronsdal embedding signature (+-----) there are compound $\mathrm{dz}$ elements, not just simple $\mathrm{dx}$ [69]. This is analogous to presence of nonradial components [27], indicating that abstract higher-dimensional quasi-geometric spatial structures can comprise several overlapped/superimposed 3D single spaces.

From traditional geometric standpoint one could argue in favor of restricting generic dimensionality to whatever can conform to the often implicit common sense requirement that if dimensions pertain to the spaces in which objects should be constructed, then they should somehow be visible too [70] or at least be unambiguously representable.

One of such arguments was offered by Möbius, who wrote that it seems remarkable that solid figures can have equality and similarity [relations] without having coincidence, while always, on the contrary, with figures in a plane or systems of points on a [straight] line equality and similarity are bound with coincidence; perhaps the reason for this may be that beyond 3D single space there is no other, no [geometric] 4D space [71]. In other words: he suggested that perhaps there is no 4D geometric space that looks just like the usual 3D single space, even though abstract quasi-geometric 4D spatial structures can exist, i.e. they can be unambiguously constructed.

Gauss apparently regarded generalizations of symmetry and congruence to geometry of $\mathrm{n}>3$ dimensions not inconsistent. Yet he did not consider the three-dimensionality of space as an inherent quality of space, but as specific peculiarity of the human mind [72] p. 177. Maybe generalized dimension is not a notion inherent to space. For one can see many quite independently varying magnitudes, i.e. abstract generic dimensions, without the necessity of any direct representation in a single quasi-geometric space, but clearly present in the actual physical reality we live in.

As it was defined in Euclidean geometry, dimensionality is not an intrinsic attribute of space but a feature of its abstract representation. For dimensionality of a given space is not really unique feature of the space, but depends upon particular choice of geometric entity for its primary/generating element [73]. Henri Cartan pointed out that even a single point with a plane passing through it can be considered as 5D space [74]. Also cube can be viewed as a square in a square and a hypercube as just a cube in a cube [75] p. 33.

Spatial perceptions are certainly conventional and so are geometric descriptions. But the conventionality does not mean that the notions of dimensionality and spatiality can be quite arbitrarily defined, however. When generic potential function $V(r)=1 / r$, for instance, is considered in pure mathematics, it does not seem to be restricted with exception of $r \neq 0$. But when the same function is viewed as representing (the root of) potential energy of an interacting radial center-bound force field, it becomes clear that the function $V(r)$ must be balanced by work done by the field on that interaction, or energy conservation law would be violated. Mathematics should be integrated with physics to avoid misconceptions.

Since abstract geometric dimensionality refers directly to formal representations of all properly constructed geometric objects (immersed in a given space), then perhaps we should try to identify first all possible classes of geometric objects (or most of them) with the algebraic structures they belong to.

If this point of view is accepted, then the $\mathrm{nD}$ hyperobject (with $\mathrm{n}>3$ ) that was often called 'space' is itself an infinite-dimensional generic abstract structure, provided this 
equating it with fact space (whose dimensionality is surely unrestricted a priori) would not create any conceptual problems or perhaps even cognitive conflicts.

By proper construction I mean relying only on rigorously proven theorems while respecting all applicable algebraic (and operational, in general) rules as well as intrinsic geometric restrictions. In the sense any arbitrary declarative statements and/or existential postulates (regardless of whether they are stated explicitly or just disguised as definitions) do not really constitute proper geometric construction.

\section{PHYSICAL ORIGINS OF THE IDEA OF TIME AS AN EXTRA DIMENSION}

The idea of time as fourth dimension was put into print by d'Alembert and Lagrange [76]. Also Poincaré observed that everything happens as if time were a fourth dimension of space [77] p. 23. That remark was upgraded by Minkowski to World Postulate, which asserts that only four-dimensional world in space and time is given by phenomena [78].

But the 4D Minkowski space is an affine space for it has no origin [79]. Since affine space is not a vector space, no point is preferred and thus its points can neither be added nor multiplied by scalars [80]. In affine geometry points, not vectors, are transformed [81]. Yet some authors still defined an abstract n-dimensional affine Minkowski space $M$ with an associated vector space $\mathrm{V}$ of translations and future timelike cone $\mathrm{C} \subset \mathrm{M}$ [82], even though the Minkowski space is just a visualization diagram convenient for plotting spacetime events [83].

Minkowski space is important for visualization of special theory of relativity (STR), for within the framework of unified trigonometry Lorentz transformations (LT) appear as just rotations in the space [84]. But it is less so for the general theory of relativity (GTR) where each freely falling observer in Minkowski spacetime has is own (local) notion of space and time $[85,86]$. Moreover, pure gravity does not really work at the classical level, whereas supergravity in $11 \mathrm{D}$, for instance, seems to work only if the spacetime part of the manifold actually is anti-de-Sitter space, not Minkowski space [87]. While surely helpful for visualization of events happening in spacetime, the Minkowski space is not a substitute for mathematical spaces and/or abstract spatial structures.

\section{GEOMETRY IS FACTUAL BUT ITS FORMALISM IS CONVENTIONAL}

Dimensionality of geometric spaces is restricted by the need for orthogonality and extended algebraic dimensionality of quasi-spatial abstract structures is contingent upon the requirement of constructability, which - in its most basic form - demands that their proper objects be operational (i.e. must not be unsolvable in principle). In the sense even though new synthetic geometry is driven by experimental facts, its formal representations adhere to conventions whose roots originated in the paradigms, which had already been espoused by the time the given representation was formulated. For paradigms lag behind the newest ideas that have been revealed by the tools the geometry had made available.

In a continuous $\mathrm{nD}$ manifold its continuity and dimensionality do not yet allow us to infer any metrical properties [which are often assumed] as properties to be ascertained by measurement; all that is known is that every point of the manifold is characterized by $n$ numbers and that to closely adjacent points closely adjacent numbers correspond [72] p. 154 . 
Although this kind of opinion is shared by many physicists, it is actually a very evasive statement that can be true only when one defines vector as an arbitrary n-tuple.

Experiments can supply hints as to what role a vector (or dimension in general) can play, but they cannot decide how a notion could be represented. Formal representation likely depends on one's expertise as well as one's depth of inquiry into the phenomena. Hence it must rely on the paradigms espoused by the authors of the given mathematical formalism, even when the guiding paradigms are not always explicitly mentioned.

Great past achievements may still have some inspirational value today, but they were great only by their contemporary standards. The Grassmann's ideas inspired geometers not only to pursue new ways to understand dimensionality and thus spaces in general, but they also tacitly discouraged their prospective attempts to tackle most inconvenient issues of geometry. Such issues were often raised by either actual- or just thought-experiments.

There are many unexplained curious experiments in exact sciences as well as thought experiments in mathematical and physical theories, of course. The most relevant issue pertaining to the realistic concept of geometric dimensionality is the unanticipated string duality that emerged in string theories [18]. When one tries to shrink a closed string by squeezing its radius beyond its minimal spatial period, the string does not crash, but the space itself seemingly reexpands in yet another dimension/direction that is quite different from all the regular spatial directions [19] p. 29 while exchanging its original radius with something that looks to me like inversed radius (or generic potential of the underlying fields interacting with the vibrating string) cast in a dual linear vector space with different and distinct basis. If my assessment of the issue is correct, this is exactly an example of what I call 'spatial inversion', which the curious behavior of Lagrange resolvents already hinted at [1]. The abstract spatial inversion, however, calls for replacement of the former paradigm of singlespace reality with that of a certain multispatial hyperspace.

Yet in order to even comprehend (not to mention understand) the whole procedure of spatial inversion one has to revamp the abstract notion of dimensionality first, and then to fathom how the entire process of inverting spaces could be accomplished. For the process to work I had envisioned an abstract hyperspatial structure that would correspond to the procedure designed to implement the process, whose presence was tentatively proposed in [38] and then briefly outlined also in [24,25]. Also a new operationally viable (as opposed to merely philosophical) approach to understanding the operational meaning of infinity and singularity is needed. These topics have been briefly discussed in $[88,89]$.

Since vectors are indeed indispensable part of the physical reality we live in, even purely algebraic treatment of them (as just n-tuples of numbers) should conform to their intended usage in physics too. However, any physical theory remains just an empty shell until we have found its reasonable physical interpretation [90] and so is any mathematical theory disconnected from the physical reality. Existential postulates can misdefine some aspects of the reality, which is factual, even if its particular formulation is conventional.

Although many authors would agree with Poincaré that all geometrical theories are conventional and that their axioms are only definitions in disguise [11] p. 50, [12] p. 293, some authors are rather uncomfortable with the fact that the, assigned by a convention, axioms could tacitly (mis-)define the abstract (and perhaps also the effective operational) reality of the chosen geometry in defiance of the actual physical reality [91].

According to Brouwer the expression of a mathematical fact is conventional, but the truth that the fact contains forces itself on our minds apart from any convention [92]. Therefore conventions must not be arbitrary, but should reflect defining characteristics of the actual physical reality. 
Notice that Poincaré's conventionalism is just a definitionary conventionalism [93]. Consequently other authors considered space as just a scaffolding and thus quite arbitrary a construct [94].

Conventionality of representing space does not mean that we should bend the truth about facts to give them a more realistic appearance, but rather that perhaps mathematics should strive to be true to the reality it attempts to describe whether it is physical or just abstract mathematical one. By 'true to reality' mathematics I mean one that is not devised just with ease of proofs in mind, but such that could synthesize its fundamental concepts from hints supplied by the curious experimental data which we neither expected nor even particularly liked. Poincaré - by divorcing space from its material content, geometry from physics - placed thus space and its geometry beyond the control of experiment [95] p.55. Yet Riemann, for instance, went in quite opposite but rather extreme direction.

\section{RIEMANN'S HIGHER DIMENSIONS AS GEOMETRIC EXTENSIONS}

From the - essentially algebraic - Grassmannian point of view, which asserts that space is only a special case of threefold extensive single linearlike magnitude, Riemann concluded that properties of geometry cannot be deduced from magnitude-idaes, but that those peculiarities through which generic geometric space distinguishes itself from other thinkable threefold extended magnitudes can be obtained only from experience [96].

While Riemann rejected the opinion that the metrical structure of space is fixed and thus not inherently independent of the physical phenomena for which it serves as just a background, he asserts that space in itself is nothing more than a 3D manifold devoid of all form and that it acquires a definite form only through the advent of a material content filling it and thus determining its metric relations [95] p. 348. This point of view allows him to just disregard any restrictions on dimensionality, because there was allegedly no spatial structure to restrict, and if the space is an abstract container that is actualized by filling it with matter, the only limitation would be the amount of matter available.

This view not only opposes artisans like Poincaré, who tried to create geometry and thus reinvent the already existing reality in his own - mathematically reductionist - conceptual image, but it also effectively denies one of the main reasons for mathematics to exist: namely to match structures to procedures or vice versa. Riemann elevated thus former mathematics' inability to grasp mathematical underpinnings of physical reality to a virtue of deference to physics, and thus subjugated mathematics to physical sciences. Yet just as matter, along with geometric structures, codefines (assumedd as empty) space so also spatial relationships codefine (along with operational procedures) matter in all its forms. Yet if the space is not empty after all, but is filled with somewhat dormant energy, then prequantum (and prerelativistic) distinction between space and energy/matter loses its previous rationale. Physics can guide pure-mathematical abstractions in conceptually meaningful directions, but it is mathematics what makes physics truly exact science.

For realists like Helmholtz the problem of space relied on the fact of existence of free moving bodies (as hinted by inferences abstracted from common experiences), but for experiential surrealists like Riemann even the [evident in common experiences] existence of freely moving [material] bodies was apparently just a hypothesis to be proved [97]. The Riemann's definition of [nonexistent] space did not solve the space problem [98]. 
To construct his geometry, however, Riemann had to throw overboard few geometric axioms, including the one which asserts that only one line can pass through two [distinct] points [11]. Yet despite this [literally patchy] character of Riemannian geometry, most physicists preferred it over the spacetime which - in its traditional interpretation - has indefinite metric [99], for Riemannian space can be seen as just a set of local tangent flat spaces [100]. The consequence of indefinite metrics of spacetime is that for some nonnull vectors their squares can be, nevertheless, zero [101], which is definitely undesirable a feature. I would say that indefinite metrics calls for disambiguation, but certainly not for evasiveness or disregarding the issues that former mathematics could not grasp or tackle.

Riemann has introduced in [96] nonhomogeneous spaces in which distance between two points in infinitely small surroundings is defined by an arbitrary (to a certain degree) differential form [102]. Riemannian space is thus a manifold similar to Euclidean space (and even to some noneuclidean spaces $[103,104])$, in which the notion of distance is conserved, but they lack homogeneity $[105,106]$. He did not resolve the issue with indefinite metric, but evaded it by postulating existence of $\mathrm{nD}$ manifolds just by analogy to the $3 \mathrm{D}$ space. Yet he gave not viable proof that all properties can be carried over from the 3D single space onto his $(n>3) D$ structures. Although the former higher-dimensional manifolds are suitable for most approximations, from a conceptual point of view they are unconceded failure of the former artlike mathematics that tacitly ignored proven results of Abel, Galois and Lagrange, among others. While approximations (and reductionism in general) are helpful for some estimates, drawing reliable inferences requires conceptually adequate (i.e. constructible and solvable, at least in principle) formal representations.

\section{ODDITIES WHEN $n>4$ DIMENSIONS ARE CAST IN A SINGLE-SPACE}

In spaces of higher dimensions functions of bounded variation may not have some of the properties they enjoy in lower dimensions [107], which often raises very annoying problems nonexistent in lower dimensions [108]. Also equations for odd and even $\mathrm{n}$ (in $\mathrm{nD}$ spaces with $\mathrm{n}>4$ dimensions) are different [109].

Extension of integrability to dimensions higher than $3^{\text {rd }}$ is also a problem because of emerging nonlocality issues, which can arise when dealing with higher-rank connections [110]. Also classification of codimension 3 singularities of vector fields is not possible for dimensions greater than $5^{\text {th }}[111]$.

On the other hand, the global existence theorem proved in more than 5 dimensions [112] is not valid for 3 dimensions [113]. These facts indicate that neither an expansion nor reduction of dimensionality preserves all features of objects immersed in the spaces. These facts defy inductive approach to dimensionality cherished in former mathematics.

Difficulty arises also when one considers symmetry of [combnatorial] dimension structure - the emphasis on orthogonal group as it were [114]. For in a 3D single space the whole bivector evidently equals to a system of $n(n-1)$ bivectors, but this is not true in $4 \mathrm{D}$ space [115] p.11. Also simple [arbitrary] extension of 4D spacetime to 5D [single] space could create some difficult problems [116].

For length and area, a central fact is that the value of the length of a curve or the area of a surface, as given by Lebesgue theory, is at least as great as that given by the classical formula, whenever the latter has actual meaning. But this is now found not to be valid in [formerly defined] higher dimensions [117], yet it holds for some other mappings [118]. 
Four is the unique dimension where a local differential equation that accounts for the radiation reaction and admitting a consistent mass-renormalization (i.e. the Dirac-Lorentz equation) exists [119]. In odd dimensions Huygens principle also does not hold, and as a result, the radiation reaction force depends on the whole past history of a charge (known as radiative tail). Yet the divergence in the tail integral can be effectively removed by the mass renormalization only in the $2+1$ theory [119]. In higher dimensions even classical theory of radiating point charges is not fully consistent [119]. This is just the tip of the proverbial iceberg of problems caused by the former, arbitrary approach to dimensions.

While abstract extensions from spaces of $(1+2) \mathrm{D}$ to $(1+3) \mathrm{D}$ are fairly simple [120], constructing higher-dimensional spatial structures was formerly done simply by analogy to 3D spaces. Theorems about hypersphere in 4D were also created just by analogy [121]. Poincaré claimed that mathematicians do not really deal in objects, but in relations among objects and so they are free to replace some objects with others so long as all the relations between them remain intact. Content to them is irrelevant - they are interested in form only [122] p. 114. But he discounted the possibility of discovery of new relations between objects and the prospect that the new relations may not be transferable to other objects.

Yet if a form is to be realistic - even if conventionally defined - it has to refer to a certain constructible structure, which in turn demands that it should also be operationally constrained by a corresponding to it viable procedure. To be viable, the procedure must not be insolvable in principle, however. For if a structure corresponds to an insolvable procedure, the structure cannot be always quite unambiguously constructed. This means that, in general, such unconstructible structure cannot exist in the actual physical reality.

It is evident that the former arbitrary approach to defining dimensions by analogy (or by induction/recursion) - [77] pp. 29, 31; [123] p. 44 - failed to ensure that indispensable generic attributes of 3D single spaces (such as no-nonsense orthogonality of dimensions) can always be carried over intact also onto higher-dimensional quasi-geometric spatial structures. But the former mathematics that relied only on transformations treated as just mappings of points from one set into/onto another set cannot ascertain transitions of other attributes of the spaces or spatial structures.

Yet existence of abstract spatial structures, such as the aforesaid 6D pangeometric hyperstructure $\mathfrak{S}$, requires also new kind of transformations that should ensure transition between its mutually dual 3D spatial structures. Such a transition does not only transform point sets, but it should also allow passage from representing geometric facts in one of the 4D spatial structures that encapsulate the 3D single spaces to its dual 4D structure and vice versa. That is why the comprehensive transition can be called spatial morphing [1].

\section{SPATIAL MORPHING AS CONCURRENT REDUCTION AND EXPANSION}

Transition of vector bases underlies morphing of one of these 3D single spaces into the other and vice versa. The 3D morphing proceeds by furling one and unfurling other 3D single space within 4D spatial structure, which is accomplishhed via spatial reduction of one of the 3D single spaces and corresponding to it spatial expansion of its dual 3D single space. This 3D spatial morphing is thus simultaneous reduction of one single space and reexpansion of its associated dual 3D single space. During spatial morphing the two inverse processess proceed in tandem. Hence the $3 \mathrm{D}$ spatial morphing must somehow relate the primary $3 \mathrm{D}$ vector basis to its dual $3 \mathrm{D}$ vector basis. 
The 4D spatial morphing is a process that proceeds on the higher, 4D pangeometric level, but it also involves the two 3D single spaces (namely the primary one and its dual) which are immersed within each of the two 4D dual spatial structures of spacetime and timespace, by transforming also each of the 4D structures into the other. The process of furling or unfurling of one of the $3 \mathrm{D}$ single spaces also causes the encompassing them $4 \mathrm{D}$ spatial structures to be morphed into their dual counterparts.

When viewed as a process, the morphing proceeds as a reduction of one 4D spatial structure via furling of its primary 3D single space, with concurrent expanding of its dual 3D single space within its dual 4D spatial structure via (presumably almost) simultaneous unfurling of the other of its 3D single spaces. If it is considered as a switch between the two 4D structures, their morphing appears as transition between their heterogenous bases.

While offering various abstract definitions of dimensions of spaces, Henri Cartan remarked that it is very difficult to demonstrate the impossibility of homeomorphism of two manifolds of different dimensions each [124,125] even though it seems impossible to establish unambiguous and continuous correspondence between points of two sets of different dimensions [126]. Besides, upon just dimensional reduction, if it would be not accompanied by dimensional expansion of its overlapping dual structure, a field theory may exhibit previously unexpected or hidden symmetries [127]. The morphing is thus a double transformation with furling of one 3D space and unfurling of its dual 3D space.

Yet this new dimensional reduction differs from the Kaluza-Klein compactification of dimensions. By analogy to bivectors, the $(1+3) \mathrm{D}=4 \mathrm{D}$ base versors $\left\{\mathrm{r}_{0}, \mathrm{r}_{1}, \mathrm{r}_{2}, \mathrm{r}_{3}\right\}$ of the 4D spacetime $\mathfrak{R}^{4}$ transform into base versors $\left\{\tau_{0}, \tau_{1}, \tau_{2}, \tau_{3}\right\}$ of its dual $(3+1) \mathrm{D}=4 \mathrm{D}$ timespace $\mathfrak{T}^{4}$ in such a way that 3 -vectors of one spatial structure transform into the extension (i.e. $4^{\text {th }}$ ) component of the other structure and vice versa $(\leftrightharpoons)$. The transformations mapp thus both: their versor-bases as well as formal representations of shapes of the objects being transformed and therefore morph also the spaces in which the objects are immersed. The transformation of the base versors themselves of the 4D structures proceeds as follows:

$$
\left\{\mathrm{r}_{1}, \mathrm{r}_{2}, \mathrm{r}_{3}\right\} \leftrightharpoons\left\{\tau_{0}\right\} \text { and }\left\{\mathrm{r}_{0}\right\} \leftrightharpoons\left\{\tau_{1}, \tau_{2}, \tau_{3}\right\}
$$

which ensures that (properly constructed) geometric objects immersed in both 4D spatial structures (spacetime and timespace) always remain fully operational and therefore their structural equations are solvable, at least in principle. In reference to physical space these 4vectors are sometimes called paravectors [128]. Notice that the schema (8), which also depicts furling and unfurling of coordinate versors, is analogous to the transformation (2).

Operationally viable structures existing in actual physical space shall provide patterns also for abstract mathematical spaces. For existence of all spatial structures is contingent upon feasibility of the operations to be performed on them. Merely postulating existence of abstract spaces without matching their structures to proper operational procedures does not really make them algebraically operational or geometrically constructible.

By stating that dimension of a vector space $\mathrm{V}$ is the maximum number of independent elements in V [129], pure mathematics trivialized the concepts of both: dimensionality and spatiality. Perhaps we should not decide what dimension "is" before we realize how it can coexist in the Nature with the other concepts intertwined with it or related to it.

If treated as science, mathematical concepts should be discovered rather than just postulated. The discovery should include its notions, structures and their operational procedures. The former artlike mathematics breeds nonsenses perpetuated for centuries [14], [31] for example, which - when applied to physical sciences - sometimes created cognitive 
conflicts and/or confusion. It adversely affected both students and researchers. Mathematics should be treated as fundamental science, not as just an abstract game of arbitrary thoughts unconstrained by any structural or operational prerequisites.

Similarly the statement: that dimension of a linear vector space is the number of its base vectors [130-132] - when generalized to higher dimensions - ignores the fact that vectorbases belong to representations of spatial structures not to spaces. Not to mention that there can be many distinct bases for representing the same spatial structure. For various vectorbases can also suggest different dimensionality [73]. I have already proposed development of a computer-aided knowledge extracting system (CAKES) to help sorting out mathematical intricacies for both students and researchers [25].

The abstract algebraic 6D hyperstructure $\mathfrak{S}$, which can also be represented as being parametrized by 6 versors spanning two distinct bases, would require some multistaged (i.e. hierarchically organized) operations in order for it to yield conceptually meaningful results. This is no longer domain of any geometry, but pangeometry.

Since vector space equipped with outer product is no longer linear [50], its spatial structures with morphing vector-bases should not be treated as just linear vector spaces.

Given the morphing schema (8) and the eqs. (3) one could tentatively envison an operational equation to aptly depict a transition between the primary and its dual vector base. Such a transition should also be described in terms of differentials, of course.

Although gradient of a point function is a tensor, it can also act as a covariant vector [133]. Having the two 4D quasi-spatial structures of spacetime and timespace, each with its own native vector base, we need a certain compound 4D gradient function that could deal with their heterogeneous vector basis.

Let us define symbolically (on Grassmannian terms) a new heterogeneous 4D nabla operator $\nabla$ as an abstract complex (rather than real) primary extension of the regular 3D nabla operator $\nabla$ via a certain new 1D curved (or imensionally furled) nabla operator $\forall$ :

$\nabla:=\nabla+\mathrm{i} \forall$

where the curved nabla $\forall$ is imaginary operator with respect to the regular nabla operator $\nabla$, for $\forall$ cannot be directly represented in terms of the primary vector basis of the space on which the regular 3D nabla operator $\nabla$ is defined. For no any other (than those three already present in the regular 3D nabla operator) distinct fourth component could ever be directly orthogonal to all the other three directional components of the 3D nabla operator.

The furled 1D nabla operator $\forall$ has thus the effect of reducing formal dimensional representation from 3D to 1D. It supports thus furling of coordinates. The regular 3D nabla operator $\nabla$ resembles thus the effect of unfurling of the coordinates by expanding formal representation of an object corresponding to a transition function from 1D to 3D formal representation of the given object in a single 3D space.

Before investigating possible consequences of the 4D operator it has to be ascertained that the proposed here relation (9) is operationally sound. This shall be done elsewhere. 


\section{CONCLUSIONS}

Dimensionality (and spatiality) is restricted by both structural and the corresponding to them operational symmetries. Abstract spatial structures endowed with higher than four dimensions (i.e. independently varying magnitudes) require hierarchically arranged operational procedures in order to meaningfully operate on them. The hierarchy of such procedures implies necessary presence of corresponding to it hierarchical structure.

This fact suggests the need for a certain abstract multispatial hyperspace to be deployed within the mathematical framework that underlies the physical reality we live in.

Consequently thus, due to inherent insolvability of general polynomial equations of degree higher than four, nontrivial cases involving dimensions higher than four require certain abstract spatial structures which would span (or perhaps should be cast) over multiple linked three-dimensional single geometric spaces in order for them to be quite unambiguously solvable, at least in principle. Hence multidimensionality with $\mathrm{n}>4$ absolutely unique abstract dimensions requires multispatiality for it to be implemented.

The multispatiality is consistent with mathematically necessary presence of two dual quasi-geometric abstract spatial structures of $(3+1) \mathrm{D}$ and $(1+3) \mathrm{D}$ kind, one of which can be identified with 4D spacetime, whereas the other with its dual 4D timespace.

It appears that duality of the two abstract spatial structures of 4D spacetime and 4D timespace implies presence of a complex, 6D algebraic structure that spans them both. This feature suggest the need for an overarching pangeometry to handle these structures.

Due to the operational - and corresponding to them structural - restrictions, presence of the 4D spatial structures of spacetime and its dual timespace requires spatial morphing, which resembles the curious behavior of Lagrange resolvents. It also mimics reexpansion of squeezed minimal string into yet another direction/dimension of the underlying spatial structure (even if it is not mentioned by name), or the field(s) immersed in the structure.

The morphing also retrodicts the strange mapping of the radius $\mathrm{R}$ (cast in spacetime) of a closed string (when it is being squeezed beyond its minimal radius) into its inverse radius $1 / \mathrm{R}$ (cast in timespace), which has already been discovered in string theories but remained mathematically unexplained thus far. The abstract mapping is consistent with specialrelativistic interpretation of physical meaning of fourth dimension of spacetime.

The furling and unfurling of the fourth dimensions (of each of these two 4D spatial structures), by which the spatial morphing is implemented, further suggest that perhaps the vibrating string is just a spacetime representation of a pointletlike energy bubble that is cast in the vector base of the 4D dual timespace structure that corresponds to the 4D spacetime. If so then perhaps the vibrating string and its dual pointletlike energy bubblet might be viewed as just two mirror images of the same underlying stringlet reflected in the spacetime and timespace, respectively. The new hyperspatial duality is analogous to the experimentally confirmed wave-particle duality that emerged in quantum mechanics.

Furthermore, if the mathematical stringlet is viewed as physically identifiable entity, then perhaps instead of looking for superpartners to regular particles in the 4D spacetime, one might view the superpartners as just abstract dual 4D representations of the regular particles, when the latter are recast in the 4D timespace that is dual to the 4D spacetime. 


\section{References}

[1] Czajko J., International Letters of Chemistry, Physics and Astronomy 17(2) (2014) 220-235.

[2] Weyl H., Das Kontinuum. Untersuchungen über die Grundlagen der Analysis. Leipzig: Verlag von Veit \& Comp., 1918, p.15.

[3] Watson A., New Sci. 118 (1988) 41-45.

[4] Tegmark M. Class. Quantum Grav. 14 (1997) L69-75.

[5] Jones J.D.S., Mysteries of four dimensions. Nature 332 (1988) 488-489.

[6] Borsuk K., Multidimensional Analytic Geometry. Warszawa: PWN, 1964, p.54 [in Polish].

[7] Stark M., Analytic Geometry with Introduction to Multidimensional Geometry. Warszawa: PWN, 1970, p.377ff [in Polish].

[8] Ivins W.M., Jr. Art \& Geometry. A study in Space Intuitions. New York: Dover, 1946, p. 102.

[9] De Vries H., Die vierte Dimension. Leipzig: 1926, p. 22.

[10] Vopěnka P., Mathematics in the alternative set theory. Leipzig: Teubner, 1979, p. 10.

[11] Poincaré H., Science and hypothesis. New York: Dover, 1952, pp. 38f, 49f, 57.

[12] Greenberg M.J., Euclidean and non-Euclidean geometries. Development and history. New York: Freeman, 1993, p.293.

[13] Heitsch W., Mathematik und Weltanschaung. Berlin: Akademie-Verlag, 1978, p. 247.

[14] Czajko J., Stud. Math. Sci. 7(2) (2013) 25-39.

[15] Czajko J., Appl. Phys. Res. 3(1) (2011) 2-7.

[16] Cartan É., Sur la structure des groupes de transformations finis et continus. Paris: Librairie Nony, 1894 [public domain reprint 390270LV00014B/256/P], p. 23.

[17] Cartan É., La topologie des groupes de Lie. Paris: Hermann, 1936, p. 7.

[18] Schwarz J.H., Spacetime duality in string theory. [pp.69-87 in: Schwarz J.H. (Ed.) Elementary particles and the universe. Cambridge: Cambridge Univ. Press, 1991].

[19] Witten E., Phys. Today April 1996, 24-30.

[20] Hausdorff F., Zwischen Chaos und Kosmos oder vom Ende der Mataphysik. BadenBaden: Agis Verlag, 1976, p.103.

[21] Dedekind R., J. reine angew. Math. 50 (1855) 272.

[22] Del Carmen Romero-Fuster M., Geometric contacts and 2-regularityof surfaces in Euclidean space. [p.307-325 in: Chéniot D. et al. (Eds.) Singularity theory. Proceedings of the 2005 Marseille Singularity School and Conference. World Scientific: Singapore, 2008, see p.309].

[23] Poincaré H., L'avenir des mathématiques. Circolo Matematico di Palermo. Atti del IV Congresso Internationale dei Matematici. Roma, 1908, see p.15.

[24] Czajko J., Chaos, Solit. Fract. 12 (2001) 951-967. 
[25] Czajko J., Chaos, Solit. Fract. 19 (2004) 479-502.

[26] DeWitt B.S., Spacetime as a sheaf of geodesics in superspace. [pp.359-374 in: Carmeli, M., Fickler, S.I. \& Witten, L. (Eds.) Relativity. New York: Plenum Press, 1970].

[27] Czajko J., Chaos, Solit. Fract. 11 (2000) 2001-2016.

[28] Czajko J., Stud. Math. Sci. 7(2) (2013) 40-54.

[29] Riemann B., Schwere, Elektricität und Magnetismus. Hannover: Carl Rümpler, 1876, p. $9 \mathrm{ff}$.

[30] Donaldson SK., Yang-Mills invariants of four-manifolds. [pp.5-40 in: Donaldson SK \& Thomas CB (Eds.) Geometry of low-dimensional manifolds:1. Gauge theory and algebraic surfaces. Cambridge: Cambridge Univ. Press, 1990, see p. 38].

[31] Czajko J., International Letters of Chemistry, Physics and Astronomy 11(2) (2014) 89-105.

[32] Mal'cev A.I., Foundations of linear algebra. San Francisco: Freeman, 1963, p. 276.

[33] Cole E.A.B., N. Cim. 40A(2) (1977) 171-180.

[34] Rauscher E.A., Lett. N. Cim. 7(10) (1973) 361-367.

[35] Bombelli L. et al. Phys. Rev. Lett. 59 (1987) 521-4.

[36] Verma R.C., Chandola, H.C. \& Rajput, B.S., Acta Cien. Indica 9 (1983) 27-30.

[37] Kim S.K., Physics: The fabric of reality. New York: Macmillan, 1975, p. 98.

[38] Czajko J., Chaos, Solit. Fract. 11 (2000) 1983-1992.

[39] Lanciani P., Found. Phys. 29(2) (1999) 251-265.

[40] Kar K.C., Ind. J. Theor. Phys. 11(3) (1963) 75-80.

[41] Kar K.C., Ind. J. Theor. Phys. 17(1) (1969) 1-11.

[42] Tifft W.G., Astrophys. Space Sci. 244 (1996) 187-210.

[43] Chudinov E.M., Mathematical problems of physics. Moscow: 1981, p. 7 [in Russian].

[44] Alvarez-Gaume L., Phys. Scripta T15 (1987) 26-33.

[45] Swamy N.V.V.J. \& Samuel M.A., Group theory made easy for scientists and engineers. New York: Wiley-Interscience, 1979, p. 72.

[46] Greider K.R., Found. Phys. 14(6) (1984) 467-505, see p. 479f.

[47] Jancewicz B., Multivectors and Clifford Algebra in Electrodynamics. Singapore: World Scientific, 1988, p. 244.

[48] Hermann R., Vector bundles in mathematical physics I. New York: Benjamin, 1970, pp. 26, 29.

[49] Grünbaum A., Geometry and chronometry in philosophical perspective. Minneapolis, MN: Univ. of Minnesota Press, 1968, p. 34.

[50] Hestenes D., Simon Stevin Quart. J. Pure Appl. Math. 62 (1988)

[51] Macdonald A., Linear and geometric algebra. Lexington, KY, 2013, p. 40.

[52] Kar K.C. \& Dutta C., Ind. J. Theor. Phys. 16(1) (1968) 1-6.

[53] Törnebohm H., A logical analysis of the theory of relativity. Stockholm: Wiksell, 1952, p. 46. 
[54] Kasner E., Am. J. Math. 43 (1921) 126.

[55] Rahula M., New problems in differential geometry. Singapore: World Scientific, 1993, p. 91.

[56] Hatzikonstantinou P. \& Moyssides P.G., Phys. Lett. A 140 (1989) 85-89, see p.88f.

[57] Teli M.T., Ind. J. Pure Appl. Phys. 23 (1985) 1-5.

[58] Cole E.A.B., Phys. Lett. 76A(5-6) (1980)157.

[59] Cole E.A.B., N. Cim. 44B (1978) 171-180.

[60] Cole E.A.B., Phys. Lett. 75A (1979) 29-30.

[61] Cocke W.J., Astrophys. Space Sci. 244 (1996) 211-218.

[62] Barashenkov V.S. \& Yur'iev M.Z., http://www.chronos.m.su.ru/seminar/ereports_of_seminar.html

[63] Arkani-Hamed N., Dimopoulos S. \& Dvali G., Phys. Lett. B429 (1998) 263-272.

[64] Antoniadis I. et al. Phys. Lett. B436 (1998) 257-263.

[65] Finkelstein D. \& Rodriguez E., Quantum time-space and gravity. [pp.247-54 in: Penrose R. and Isham C.J. (Eds.) Quantum concepts in space and time. Oxford: Clarendon Press, 1986].

[66] Thorne K.S., Black holes and time warps. New York: W.W. Norton, 1994, p. 128.

[67] Kasner E., Trans. AMS 27 (1925) 101-105, see p. 104.

[68] Kasner E., Am. J. Math. 43(2) (1921) 130-133.

[69] Fujitani T., Ikeda M. \& Matsumoto M., J. Math. Kyoto Univ. 1-1 (1961) 43-61.

[70] Freund P.G.O., Physica 15D (1985) 263-269.

[71] Möbius., On higher space. [in excerpts from “Der barycentrische Calcul.” Leipzig, 1827, part 2 ch. 1 - pp.525-526 in: Smith D.E.A (Ed.) Source book in mathematics II. New York: Dover, 1959, see p.5 26].

[72] Jammer M., Concepts of space. The history of theories of space in physics. New York: Dover, 1993, pp. 154, 173, 177.

[73] Keyser C.J., The human worth of rigorous thinking. Essays and addresses. New York: Columbia Univ. Press, 1916, p. 106.

[74] Cartan H., Les espaces métriques fondés sur la notion d'aire. Paris: Hermann, 1933, p.7.

[75] Rucker R., The fourth dimension. Toward a geometry of higher reality. Boston: Houghton Mifflin, 1984, p. 202.

[76] Cajori F., Am. Math. Month. 33 (1926) 397.

[77] Poincaré H., Mathematics and science. Last essays. New York: Dover, 1963, pp. 17, 28.

[78] Minkowski H., Space and time. [pp.75-91 in: Lorentz H.A. et al. The Principle of Relativity. New York: Dover, 1923, see p. 83].

[79] Deligne P., Freed D.S., Supersolutions. [pp.227-355 in: Deligne P. et al. Quantum Fields and Strings: A Course for Mathematicians I. Providence, RI: AMS, 1999, see p. 231].

[80] Snapper E., Troyer R.J., Metric Affine Geometry. New York: Dover, 1989, p. 59.

[81] Hausner M., A vector approach to geometry. York: Dover, 1998, p. 330. 
[82] Deligne P., Freed D.S., Sign Manifesto. [pp.357-363 in: Deligne P. et al. Quantum Fields and Strings: A Course for Mathematicians I. Providence, RI: AMS, 1999, see p. 358].

[83] Martin L., General relativity. A guide to its consequences for gravity and cosmology. New York: Ellis, Harwood, 1988, p. 20.

[84] Jaccarini A., Can. J. Phys. 51 (1973) 1304-1312.

[85] Geroch R., General relativity from A to B. Chicago: The Univ. of Chicago Press, 1978, pp. 166, 171.

[86] O’Neill B., Semi-Riemannian geometry with applications to relativity. New York: Academic Press, 1983, p. 171.

[87] Chodos A., Comments Nucl. Part. Phys. 13 (1984) 171-81.

[88] Czajko J., Chaos, Solit. Fract. 21 (2004) 261-271.

[89] Czajko J., Chaos, Solit. Fract. 21 (2004) 501-512.

[90] Bergmann P.G., Trans. NY Ac. Sci. 38 (1977) 1.

[91] Mooij J.J.A., Synthese 16 (1966) 53.

[92] Ambrose A., A controversy in the logic of mathematics. Univ. of Wisconsin Press [reprinted from "The Philosophical Review" of Nov' 1933].

[93] Diederich W., Konventionalität in der Physik. Wissenschaftstheoretische Untersuchungen zur Konventionalismus. Berlin, 1974, pp. 16, 22.

[94] Milne E.A., Relativity, gravitation, and world-structure. Oxford: Clarendon Press, 1935, p. 289.

[95] D’Abro A., The evolution of scientific thought. From Newton to Einstein. New York: Dover, 1950, pp. 55, 348.

[96] Riemann B. Ueber die Hypothesen, welche der Geometrie zu Grunde liegen. pp. 272282 [in: Weber H. (Ed.) Bernhard Riemann's gesammelte mathematische Werke. New York: Dover, 1953].

[97] Freudenthal H., Im Umkreis der sogenannten Raumprobleme. [pp. 322-327 in: BarHillel Y. et al. (Eds.) Essays on the foundations of mathematics. Jerusalem: At the Magnus Press, 1961].

[98] Reichenbach H., Gesammelte Werke III: Die philosophische Bedeutung der Relativitätstheorie. Braunschweig: Vieweg, 1979, p. 394.

[99] Esposito G., Quantum gravity, quantum cosmology and Lorentzian geometries. Berlin: Springer, 1992, p. 230.

[100] Band W., Phys. Rev. 61 (1942) 702-707.

[101] Laurent B., Introduction to spacetime. Singapore: World Scientific, 1994, p. 32.

[102] Cartan E., La théorie des groupes et les recherches récentes de géométrie différentielle. [pp.891-904 in: Cartan E. Euvres complètes. Part 3.1. Paris: Éditions du CNRS, 1984, see p. 892]. 
[103] Cartan, E., Leçons sur la géométrie projective complexe. Paris: Gauthier-Villars, 1950, p. 187.

[104] Cartan E., L'axiome du plan et la géométrie différentielle métrique. [pp. 1057-1065 in: Cartan, E. Euvres complètes p.III v.2. Paris: CNRS, 1984, see p. 1058].

[105] Cartan E., Les récents généralisations de la notion d'espace. [pp. 863-889 in: Cartan E. CEuvres complètes. Part 3.1. Paris: Éditions du CNRS, 1984, p. 864].

[106] Pierpont J., Am. Math. Month. 30 (1923) 425.

[107] Gora P. \& Boyarsky A., Am. Math. Mon. 99 (1992) 159.

[108] Chung D.J.H., Kolb E.W. \& Riotto A., arXiv:hep-ph/0008126 14-Aug-2000.

[109] Friedlander F.G., The wave equation on a curved spacetime. Cambridge: Cambridge Univ. Press, 1975, p. $228 f$.

[110] Ferreira L.A., Leite E.E. Nucl. Phys. B 547 (1999) 471-500.

[111] Takens F., IHES Publ. Math. 43 (1973) 47-100.

[112] Klainerman S.L., Commun. Pure Appl. Math. 33 (1980) 43-101.

[113] John F., PNAS USA 77(4) (1980) 1759-1760.

[114] Bastin K. \& Kilmister C.W., Combinatorial physics. Singapore: World Scientific, 1995, p. 149.

[115] Cartan E., Leçons sur la géométrie des espaces de Riemann. Paris: Gauthier-Villars, 1963, pp. 11, 14ff.

[116] Reichenbächer E., Phys. Z. 29 (1928) 908-911.

[117] Goffman C., PNAS USA 63 (1969) 38-39.

[118] Goffman C. \& Ziemer W.P., PNAS USA 65 (1970) 491-4.

[119] Gal'tsov D.V., (2004) http://arxiv.org/PS_cache/hep-th/pdf/0112/0112110.pdf .

[120] Coxeter H.S.M., Am. Math. Month. 50 (1943) 217.

[121] Poincaré H., C.R. Acad. Sci. Paris 140 (1906) 113.

[122] Nicholson W.K., Introduction to abstract algebra. New York: Wiley, 1999, pp. 79, 512 \& 111.

[123] Poincaré, H., The value of science. New York: Dover, 1958, p. 54.

[124] Cartan H., Sur la notion de dimension. [pp.1414-1425 in: Remmert R. \& Serre J.-P. (Eds.) Cartan H. Oeuvres. Collected works III. Berlin: Springer-Verlag, 1979, see p. 1415].

[125] Lebesgue H., Math. Ann. 70 (1911) 166.

[126] Lebesgue H., Fund. Math. 2 (1921) 256, see p. 265.

[127] De Wit B. \& Van Proeyen A., Int. J. Mod. Phys. 3 (1994) 31-47.

[128] Baylis W.E. \& Sobczyk G., J. Theor. Phys. 43(10) (2004) 2061-2079.

[129] Artin E., Galois theory. Notre Dame, IN: 1959, p. 4.

[130] Smiley M.F., Algebra of matrices. Boston: Allyn \& Bacon, 1965, p. 87. 
[131] Curtis C.W., Linear algebra. An introductory approach. Boston: Allyn \& Bacon, 1968, p. 37.

[132] Berberian S.K., Introduction to Hilbert space. New York: Oxford Univ. Press, 1961, p. 23.

[133] Cartan E., Leçons sur la théorie des espaces a connexion projective. Paris: GauthierVillars, 1937, p. 216. 